\title{
Lineage composition and trichothecene production of Gibberella zeae population in Korea
}

\author{
Yin-Won LeE ${ }^{* 1}$, Jae-Jin JEON ${ }^{* 1}$, Hun KIM ${ }^{* 1}$, In-Young JANG ${ }^{* 1}$, \\ Hye-Sun $\mathrm{KIM}^{* 1}$, Sung-Hwan YUN ${ }^{* 2}$ and Jeon-Gon $\mathrm{KIM}^{* 3}$ \\ ${ }^{* 1}$ School of Agricultural Biotechnology and Center for Agricultural Bio-materials, Seoul National \\ University \\ (Seoul 151-742, Korea) \\ *2 Division of Life Sciences, Soonchunhyang University \\ (Asan 336-745, Korea) \\ *3 National Crop Experiment Station \\ (Suwon 441-100, Korea)
}

\begin{abstract}
Summary
Gibberella zeae (anamorph: Fusarium graminearum) is an important pathogen of cereal crops in many areas of the world. Infected grain with this fungus is often contaminated with mycotoxins. Phylogenetic trees of G. zeae isolates from maize and rice were constructed by using amplified fragment length polymorphism (AFLP). AFLP showed polymorphic bands and the bands, haplotypic loci, were used to analyze genetic diversity of $G$. zeae population. Population structure of maize isolates consisted of four lineages. Lineage 7 was the major group followed by lineage 3, lineage 6, and lineage 2 . On the other hand, the population structure of rice isolates consisted of only lineage 6. Maximum parsimony trees based on sequencing data from Tri101, Tri7 and MAT-1-1 genes were found to be concordant with AFLP data. Trichothecene production of maize isolates was variable; lineage 7 and 3 isolates were deoxynivalenol (DON) producers and most lineage 6 and 2 isolates were nivalenol (NIV) producers. All lineage 6 isolates from rice were mostly NIV producers with a few DON chemotypes. This result suggests that genetic variations exist in G. zeae population from maize and rice in Korea.
\end{abstract}

Key words : Gibberella zeae, lineage, trichothecenes, corn, rice.

\section{Introduction}

Gibberella zeae (Schwein.) Petch (anamorph: Fusarium graminearum Schwabe) is an important pathogen of cereals including maize, wheat and barley ${ }^{1)}$. Head blight and ear rot reduce grain yield, and harvested grain is contaminated with mycotoxins, such as 
trichothecenes and zearalenone.

G. zeae may be divided into two chemotaxonomic groups based on production of 8ketotrichothecenes ${ }^{2}$. The deoxynivalenol (DON) chemotype produces DON and acetylDONs such as 3-acetyl-DON and 15-acetyl-DON. The nivalenol (NIV) chemotype produces NIV and 4-acetyl-NIV (fusarenone-X). DON and NIV are often found in cereals harvested in Korea and Japan ${ }^{3.4}$. NIV is present at higher levels than DON in cereals from these countries.

G. zeae is homothallic and may produce abundant perithecia in the field. Bowden and Leslie ${ }^{5)}$ suggested that the highly genotypic diversity of $F$. graminearum could be due to occasional outcrossing and developed crossing methods in laboratory conditions. Recently, O'Donnell et al. ${ }^{6}{ }^{6}$ used DNA sequences of six single-copy genes to resolve worldwide collected $F$. graminearum strains into seven distinct phylogenetic lineages. Only lineage VII has been found within North American field collections ${ }^{7)}$, whereas lineage VI were Asian isolates. Gale et al. ${ }^{8)}$ also reported that all isolates from wheat fields in eastern China belonged to lineage VI.

Collections of Fusarium isolates, mainly $F$. graminearum, from maize and barley have been examined for mycotoxin production ${ }^{9)} . F$. graminearum occurs in Korea as DON- and NIV-producing chemotypes; DON chemotypes are predominant in maize isolates and NIV chemotypes are predominant in barley and rice isolates. In this study, we wanted to determine if the known lineages were distributed in Korea and if the lineages are associated with fertility and toxin production. Such information could help resistance breeding strategies and plant quarantines. More detailed understanding of population structure could also provide clues to the evolutionary behavior of this organism.

Our objectives in this study were (i) to determine the lineages of $F$. graminearum populations in Korea, (ii) to test fertility of the isolates, (iii) to study mycotoxin production by the isolates, and (iv) to determine the relationship between the population structures and some phenotypes including fertility and toxin production.

\section{Materials and Methods}

Fungal isolates Diseased maize ears and rice seeds were randomly collected in 19992001 from farmers' fields in Gangwon province and in southern provinces of Korea, respectively. One hundred seeds of each sample were soaked in $2 \%$ sodium hypochlorite for $2 \mathrm{~min}$, rinsed in sterile water, transferred to potato dextrose agar (PDA, Difco Laboratories, Detroit, MI, USA), and incubated at $25{ }^{\circ} \mathrm{C}$ for 4-7 days. Fusarium isolates, with carmine-red pigmentation, were transferred to home-made PDA (20\% potato extract, $2 \%$ dextrose, agar $1.5 \%$ ), carnation leaf agar or both, and incubated under fluorescent lamps (cool white type, 5,000 lux) at $25{ }^{\circ} \mathrm{C}$. We recovered 584 isolates and 
269 of $F$. graminearum from the maize and rice, respectively. All cultures were purified by single-spore isolations and stored as spore suspensions in $15 \%$ glycerol at $-80{ }^{\circ} \mathrm{C}$. Isolates were maintained for short periods on slants of PDA as needed. Six hundred and six isolates of $F$. graminearum were used in AFLP fingerprinting with additional 19 isolates from the United States. In addition, F. pseudograminearum, F. sporotrichioides, $F$. culmorum, and $F$. crookwellense were included for comparison with $F$. graminearum. DNA isolation DNA was isolated by a cetyltrimethyl ammonium bromide (CTAB) procedure $^{10)}$. Samples and sample dilutions were run in $1 \%$ agarose gels containing TAE (40 mM Tris-acetate, $1 \mathrm{mM}$ EDTA, pH 8.0) and $0.5 \mu \mathrm{g} / \mathrm{ml}$ ethidium bromide. DNA yields ranged from $100-1000 \mu \mathrm{g}$ of DNA per culture. The concentration of each DNA sample was adjusted to $20 \mu \mathrm{g} / \mathrm{ml}$ for use in AFLP analysis.

$\boldsymbol{A F L P}$ AFLPs were generated as described by Zeller et al. ${ }^{7)}$ for all isolates with the specific primer pair combinations Eco+AA/Mse+AT, Eco+CC/Mse +CG, and $E c o+T G / M s e+T T$. The $E c o$ RI primers in the final specific amplification reactions were 5' end-labeled with $\left[\gamma^{33}\right.$ P]ATP (NEN Life Sciences, Boston, MA, USA). AFLP fragments were separated in $6 \%$ denaturing polyacrylamide gels in 1x TBE buffer (100 $\mathrm{mM}$ Tris base, $100 \mathrm{mM}$ boric acid, and $2 \mathrm{mM}$ EDTA, $\mathrm{pH} \mathrm{8.0)} \mathrm{in} \mathrm{both} \mathrm{gels} \mathrm{and} \mathrm{running}$ buffer. Dried gels were exposed to X-ray film for 2-5 days at room temperature to identify DNA bands. We manually scored polymorphic AFLP bands ranging from 100 to $800 \mathrm{bp}$ in length.

Analysis of AFLP data To analyze AFLP profiles, manual scoring of the presence or absence of bands across the set of maize and rice isolates were done for each of the 3primer pair combinations. It was assumed that bands of the same molecular size in different individuals were identical. Each band was treated as a single independent locus with two alleles and unresolved bands or missing data were scored as ambiguous. Examination of isolate relatedness was done with the Unweighted Pair Grouping by Mathematical Average (UPGMA). UPGMA was performed in PAUP 4.0 beta $8^{11)}$, and booth trap analyses (1,000 iterations) were conducted on the resulting UPGMA to support for any resulting group.

Fertility test Each isolate was inoculated on carrot agar and incubated at $25{ }^{\circ} \mathrm{C}$ under fluorescent lamps with a 12-hour photoperiod. Plates were arranged right up side in a single layer on the incubator shelves. On day 7, $1 \mathrm{ml}$ of sterile Tween 60 (2.5\%) solution was added to each plate. Aerial mycelia were knocked down with a sterile bent glass rod while plates were rotated several times to spread the solution. Plates were returned to the incubator. Perithecia and ascospores were observed under microscope after 7 days. Each isolate was tested at least twice to confirm fertility.

Toxin analysis Each isolate was screened for trichothecene production on rice medium. Rice cultures were harvested after 3 weeks of incubation at $25^{\circ} \mathrm{C}$ and extracted by a procedure reported previously ${ }^{9}$. A portion of each extract was reacted with 
trimethylsilylating reagent and analyzed with a Shimadzu QP-5000 gas chromatographmass spectrometer (GC-MS) as reported previously ${ }^{9}$.

Inoculation The two representative isolates (lineage 7 and 6) were co-inoculated with the same concentrations of conidia $\left(1 \times 10^{5}\right.$ conidia/ml) to rice (cv. Nakdong) at flowering stage and overwintered. The perithecia on rice straw were then randomly collected and ascospores were single-spored. PCR was performed with Tri7 and Tril3 in order to differentiate the two lineages.

\section{Results and Discussion}

AFLP analysis AFLP analysis was conducted on DNA prepared from all 853 isolates of $F$. graminearum using three primer pairs. The number of DNA fragments detected varied with the primer pair. With the three primer pairs combined, a total of 204 AFLP bands were recorded across all 853 isolates. Among these AFLP bands, 187 were polymorphic. Of the primer combinations, ECCMCG combinations revealed the lowest number of bands and highest levels of polymorphism.

UPGMA clustering analysis was carried out in order to demonstrate the genetic similarities among the $853 \mathrm{~F}$. graminearum isolates together with 17 U.S. isolates. The dendrogram separated the isolates into four groups in the maize population and one group in the rice population. Other species including $F$. culmorum, $F$. crookwellense, $F$. pseudograminearum, and $F$. sporotrichioides were significant outliers from the $F$. graminearum groups. Lineage compositions of the maize population and rice population were quite different. The maize population consisted of 4 lineages, which were lineage 7 , 6,3 , and 2 . On the other hand, the rice population consisted of a sole lineage, which was lineage 6 . This result was also confirmed by MP analysis of the combined sequence data including Tri101, Tri7, and MAT-1 genes (data not shown).

Trichothecene production was variable to each lineage. In the maize population, the lineage 7 isolates and lineage 3 isolates were DON chemotypes whereas lineage 6 isolates except one and lineage 2 isolates were NIV chemotypes (Table 1). The toxin concentrations of lineage 3 and 2 isolates were relatively lower than those of lineage 7 and 6 isolates. Polymorphism of Tri7 and Tril3 in PCR assay was observed in each lineage. Lineage 7 isolates showed DON-specific bands and lineage 6 isolates except a few showed NIV-specific bands. All lineage 3 isolates showed that Tri7 was NIVspecific and Tril3 was DON-specific.

On the other hand, most rice isolates were NIV chemotypes and 9 out of 269 isolates were DON chemotypes. Most isolates showed that both Tri7 and Tril3 were NIV-specific polymorphism, although some isolates were variable (Table 2). Two isolates showed that Tri7 and Tril3 was NIV-specific band and was DON-specific band, respectively. Among the 9 isolates of DON chemotypes, 7 isolates co-produced DON 
Table 1. Lineage composition and trichothecene production of Fusarium graminearum population from maize in Gangwon province of Korea.

\begin{tabular}{|c|c|c|c|c|c|}
\hline \multirow{2}{*}{ Lineage } & \multirow{2}{*}{ No. of isolates } & \multicolumn{2}{|c|}{ PCR } & \multirow{2}{*}{$\begin{array}{c}\text { Chemical } \\
\text { analysis }\end{array}$} & \multirow{2}{*}{ Fertility(\%) } \\
\hline & & Tri7 & Tril3 & & \\
\hline \multirow{2}{*}{7} & 399 & DON & DON & DON & \multirow{2}{*}{100} \\
\hline & 31 & DON & DON & $\mathrm{nd}^{\mathrm{a}}$ & \\
\hline \multirow{4}{*}{6} & 53 & NIV & NIV & NIV & \multirow{4}{*}{69} \\
\hline & 17 & NIV & NIV & $\mathrm{nd}^{\mathrm{a}}$ & \\
\hline & 1 & DON & NIV & NIV & \\
\hline & 1 & DON & DON & DON & \\
\hline \multirow{2}{*}{3} & 43 & NIV & DON & DON & \multirow{2}{*}{25} \\
\hline & 32 & NIV & DON & $\mathrm{nd}^{\mathrm{a}}$ & \\
\hline \multirow{2}{*}{2} & 2 & NIV & NIV & NIV & \multirow{2}{*}{14} \\
\hline & 5 & NIV & NIV & $\mathrm{nd}^{\mathrm{a}}$ & \\
\hline Total & 584 & & \multicolumn{3}{|c|}{ DON 442 / NIV $56 /$ nd $^{\text {a }} 86$} \\
\hline
\end{tabular}

a not detected

Table 2. Lineage composition and trichothecene production of Fusarium graminearum population from rice in southern provinces of Korea.

\begin{tabular}{|c|c|c|c|c|c|}
\hline \multirow{2}{*}{ Lineage } & \multirow{2}{*}{ No. of isolates } & \multicolumn{2}{|c|}{ PCR } & \multirow{2}{*}{$\begin{array}{c}\text { Chemical } \\
\text { analysis }\end{array}$} & \multirow{2}{*}{ Fertility $(\%)$} \\
\hline & & Tri7 & Tril3 & & \\
\hline 6 & 260 & NIV & NIV & NIV & 36 \\
\hline 6 & 1 & NIV & DON & DON & 100 \\
\hline 6 & 1 & NIV & DON & $\mathrm{nd}^{\mathrm{b}}$ & 0 \\
\hline 6 & 7 & $-{ }^{a}$ & DON & DON & 86 \\
\hline Total & 269 & & & & 37 \\
\hline
\end{tabular}

a not amplified

b not detected

and 3-acetyl-DON. Since 3-acetyl-DON producers were defected $\operatorname{Tr} i 7^{12)}$, we could not amplify Tri7 in these isolates.

In this study, we found that lineage composition was quite different between Gangwon province and southern provinces in Korea. There were some close associations between lineage composition and toxin production. O'Donnell et al. ${ }^{6}$ and Gale et al. ${ }^{8)}$ reported that Asian isolates of $F$. graminearum belonged to lineage 6 . The members of the $F$. graminearum species complex, consisting of 4 lineages, were the primary etiological agents of maize ear rot in Korea. The occurrence of lineage 3 isolates is also 
characteristic since they belong to the African clade ${ }^{6)}$.

There was a remarkable difference in fertility among the lineages when the isolates were grown on carrot medium. The fertility of lineage 7 isolates was $100 \%$ followed by lineage 6 , lineage 3 , and lineage 2 .

The main question arising from this study is why the different lineages of $F$. graminearum are distributed in the two regions. To address this problem, we briefly looked into $F$. graminearum isolates, which were obtained from rice fields next to maize fields in Gangwon province and approximately $80 \%$ of the isolates were lineage 6 followed by lineage 7 . Therefore we co-inoculated the representative isolates of lineage 7 and 6 to rice at flowering stage. After inoculation, the rice plants were left over during the winter. The perithecia on rice straw were randomly collected and one ascosopre was selected from each perithecium. A total of 247 isolates were recovered and subjected to PCR assay in order to differentiate DON-producing and NIV-producing isolates. NIV/DON chemotypes were recovered at 7:1 ratio when the two lineages were coinoculated to rice. This result suggests that rice may be a selection pressure to $F$. graminearum population and confer better environment for survival to lineage 6 . Currently we are doing inoculation tests to rice with some other isolates.

\section{References}

1) Marasas, W.F.O., Nelson, P.E., Toussoun, T.A.: Toxigenic Fusarium species, Identity and Mycotoxicology, (1984), The Pennsylvania State University Press, University Park, USA

2) Ichinoe, M., Kurata, H., Sugiura, Y., Ueno, Y.: Appl. Environ. Microbiol., 46, 1364-1369 (1983)

3) Kim, J.-C., Kang, H.-J., Lee, D.-H., Lee, Y.-W., Yoshizawa, T.: Appl. Environ. Microbiol. 59, 3798-3802 (1993)

4) Yoshizawa, T., Jin, Y.Z.: Food Addit. Contam., 12, 689-694 (1995)

5) Bowden, R.L., Leslie, J.F.: Phytopathology, 89, 182-188 (1999)

6) O'Donnell, K., Kistler, H.C., Tacke, B.K., Casper, H.H.: PNAS, 97, 7905-7910 (2000)

7) Zeller, K.A., Bowden, R.L., Leslie J.F.: Phytopathology, 93, 874-880 (2003)

8) Gale, L.R., Chen, L.-F., Hernick, C.A., Takamura, K., Kistler, H.C.: Phytopathology, 92, 13151322 (2002)

9) Seo, J.-A., Kim, J.-C., Lee, D.-H., Lee, Y.-W.: Mycopathologia, 134, 31 -37 (1996)

10) Kerenyi, Z., Zeller, K.A., Hornok, L., Leslie, J.F.: Appl. Environ. Microbiol., 65, 4071-4076 (1999)

11) Swofford, D.L.: PAUP: Phylogenetic Analysis Using Parsimony and Other Methods, (1998), Sinauer Associates, Sundurland, MA, USA

12) Kimura, M., Tokai, T., O’Donnell, K., Ward, T. J., Fujimura, M., Hamamoto, H. Shibata, T., Yamaguchi, I.:FEBS Lett., 539, 105-110 (2003) 